\section{Aproximaciones \\ a la función social de la argumentación en la escuela rural. Una mirada sociocultural}

Approaches to the social role of argumentation in the rural school. A sociocultural look
Pedro R. Guerra Meza'

pedroguerra.527@yahoo.es

https://doi.org/10.22209/rhs.v7n1a03

Recibido: marzo 26 de 2019.

Aceptado: junio 7 de 2019

\section{Resumen}

Las prácticas de enseñanza de la argumentación deberían ser un espacio interactivo que le permita al docente explorar todas las posibilidades comunicativas del estudiante. Se podrían tejer conglomeradas rutas de abarcamiento pedagógico y didáctico correspondientes a las diferentes situaciones comunicativas auténticas que traen de su contexto natural y que ellos discurren en el salón de clases y en todos los espacios escolares. Es un tipo de discurso que tiene presencia en su cotidianidad, lo cual permite su interacción. En este sentido, es la escuela la encargada de formalizarlos para que adquieran sentido dentro del universo de la comunicación. Con este apartado investigativo, que tuvo como objetivo comprender las prácticas de

1 Docente de la Universidad de Antioquia, Facultad de Educación, Medellín, Colombia; Docente de la Universidad de Córdoba, Facultad de Educación, Montería, Colombia; Docente- tutor del Programa Todos a Aprender en el departamento de Antioquia, Subregión de Urabá, Ministerio de Educación Nacional. enseñanza de la argumentación en El Centro Educativo Rural Montecristo desde una perspectiva sociocultural, empleando una metodología cualitativa, dando como resultado una discusión en torno a una función social de la argumentación, se sugiere que la escuela debería comenzar a estructurar sus programas curriculares hacia propuestas de trabajo pedagógico que privilegien el proceso formativo de la argumentación en sus estudiantes. Es decir, un acercamiento a esa función social en donde se les reconozcan sus discursos y se sientan partícipes con sus enunciaciones de decisiones políticas dentro de su contexto comunitario.

Palabras clave: Carácter social; Educación; Enseñanza; Prácticas argumentativas; Ruralidad.

\section{Abstract}

Argumentative teaching practices should be an interactive space allowing the teacher to explore all the communicative possibilities of students. Clusters of pedagogical and didactic roadmaps could be interconnected which are aligned to the different authentic communicative situations that students bring from their natural context and about which they can reflect both in the classroom and in all school environments. This type of discourse is present in students' daily lives, which promotes their interaction. In this regard, the school is tasked with formalizing such discourse so that it becomes meaningful within the communication universe. This research study 
aimed to understand, from a sociocultural perspective, the argumentative teaching practices in El Centro Educativo Rural Montecristo, by means of a qualitative methodology, resulting in a discussion around the social role of argumentation. It is suggested that the school should begin to structure its curricular programs around pedagogical proposals that privilege an argumentative training process among its students. That is to say, to implement an approach to that social role that acknowledges students' discourse and under which they feel engaged through statements of political decisions within their community context.

Keywords: Social character; education; teaching; argumentative practices; rurality.

\section{Introducción}

$\mathrm{E}$ I presente artículo hace parte del cuarto capítulo de la investigación «Las prácticas de enseñanza de la argumentación desde un enfoque sociocultural, un acercamiento a la educación básica primaria en la ruralidad» realizada en el Centro Educativo Rural Montecristo, municipio de San Juan de Urabá, Antioquia entre los años 2014 y 2015, bajo el escenario de estudio de maestría con la Universidad de Antioquia.

En relación, profundizar en el contexto investigativo de la argumentación es adentrar en un campo que tiene varias perspectivas de análisis, entre ellas: la retórica y la lógica. Desde la perspectiva retórica, la argumentación se asocia con un conjunto de estrategias que organizan un discurso persuasivo. Desde la perspectiva lógica, la argumentación es un tipo de razonamiento que se emparenta con la demostración.
La modalidad de la argumentación es un área que ha llamado mucho el interés de los investigadores en la última década. Se encuentran trabajos sobre este tema en el derecho, como es el caso del libro La argumentación en el Derecho publicado por Gascón y García, en el año 2015²; en el campo de la lingüística, como lo es el trabajo de Mendoza, artículo publicado en el año 2015, titulado La validez en los exámenes de alto impacto. Un enfoque desde la lógica argumentativa ${ }^{3}$, y, desde luego, en la educación, en especial en la enseñanza de la lengua y la literatura. Aquí se trae a colación la investigación realizada por Ochoa en el año 2005, titulada Estrategias didácticas para el desarrollo de argumentación oral en quinto grado de educación básica colombiana4.

De acuerdo con esta última parte, la relacionada con la educación, es en el municipio de San Juan de Urabá, mediante el acompañamiento pedagógico a docentes en las aulas de clases y en el compartir experiencias de tipo pedagógicas y didácticas a través de comunidades de

2 En este libro se tratan las múltiples dimensiones que confluyen en el estudio del razonamiento jurídico, situando la teoría de la argumentación en dos contextos diversos, uno jurídico-político y el contexto teórico en el que esta disciplina ha debido afrontar el problema de la racionalidad como el resto de las ciencias sociales.

3 En el presente artículo se expone la importancia de la validez a partir de la segunda mitad del siglo XX, las dificultades latentes al momento de validar exámenes a finales del siglo pasado y, posteriormente, se presenta una novedosa concepción de abordar la validez en la evaluación educativa. Vista desde la lógica de la argumentación, la validez consiste en todo un proceso de inferencias que deben encontrarse sustentadas en la evidencia y en el respaldo de las mismas. Este modelo para validar exámenes desde la lógica argumentativa se está siguiendo en diversas áreas educativas a nivel internacional; sin embargo, ha cobrado especial relevancia en la evaluación de segundas lenguas.

4 En este trabajo la autora diseña, implementa y evalúa una estrategia didáctica para permitir el desarrollo de la argumentación oral. 
aprendizajes y sesiones de trabajo situado, en función del programa Todos a Aprender ${ }^{5}$ del Ministerio de Educación Nacional (MEN), en donde se configura el interés por desarrollar una propuesta de investigación sobre la enseñanza de la argumentación en la escuela y, más precisamente, en un contexto rural. En los mencionados encuentros se concluye que una de las causas relacionadas con la poca apropiación de la argumentación por parte de los niños en la Educación Básica Primaria, y que suele cobrar fuerza en entornos rurales, es la relacionada con las prácticas de enseñanza llevadas a cabo por los docentes, las cuales al parecer, no se armonizan con el contexto sociocultural. Estas se deben pensar, más bien, como acciones educativas que giren en torno a la participación social, a posturas críticas y reflexivas en cuanto a la economía de la región, la idiosincrasia, la población o los servicios públicos, entre otros factores que son propios del contexto. Además, la argumentación como una práctica inserta en la vida, sirve para participar de la cultura y de esa dinámica social.

En consideración, aquí se expone una conceptualización acerca de la función social de la argumentación en la educación básica rural desde las consideraciones de un enfoque sociocultural. Para ello, se habla del carácter social de la escuela rural como un espacio sugestivo en donde se da el acto de enseñar y se llevan a cabo múltiples determinaciones, interacciones, intereses y expectativas que se nutren a diario

5 Es un programa del MEN que busca promover la calidad de la educación y disminuir las brechas entre lo urbano y lo rural, de modo que los estudiantes de las zonas rurales y urbanas, tengan las mismas oportunidades para el desarrollo integral de sus competencias, mediante la cualificación del ejercicio profesional de los docentes y el fortalecimiento organizacional de las instituciones educativas. en el diálogo constante de sus actores. Por estas razones, la escuela representa un espacio propicio para llevar a cabo prácticas argumentativas, ya que estas nacen de la interacción de las personas y establecen, entre otras cosas, acuerdos, diálogos o confrontan ideas.

Esta investigación tuvo una intención descriptiva y analítica, pues en ella se reconstruye el concepto de argumentación en la ruralidad. Es a partir de esta dinámica en donde se plantea una discusión en torno a su función social, la cual, muchas veces, pasa desapercibida en las aulas de clases.

\section{Materiales y métodos}

a. Tipo de estudio: se realizó un estudio cualitativo de la información recolectada, la población objeto de estudio fueron los docentes del Centro Educativo Rural Montecristo, ubicado en el municipio de San Juan de Urabá, departamento de Antioquia, subregión de Urabá; el tamaño de la muestra fueron los cinco docentes que atienden la básica primaria en este establecimiento educativo, razón por la cual, el tipo de muestreo, no aleatorio, fue el muestreo por conveniencia.

b. Método de investigación: el método de investigación escogido fue la etnográfica escolar, el cual va en sintonía con el enfoque cualitativo debido a su relación con la realidad social, en este caso la escuela, a través de diferentes herramientas, tales como la observación participante y los grupos de discusión. En cuanto a la observación participante, se le pidió a los docentes que abordaran el tema de la argumentación en las clases a observar. Se registró la información 
de este proceso en el diario de campo y al final de cada clase se hizo una reflexión con cada docente. En lo respectivo a los grupos de discusión, que en total fueron tres, la discusión giró en torno a ejes temáticos definidos con anterioridad: la concepción de argumentacion de cada participante, las condiciones de la enseñanza en la ruralidad, los recursos disponibles, vinculación de las familias, la finalidad y función social de la enseñanza. Todo ello enfocado al campo de la argumentación. Estas herramientas señaladas, aportaron información detallada para el tratamiento sistemático e investigativo de la información recolectada y ello permitió el surgimiento de una categoría para la práctica de enseñanza de la argumentación en la ruralidad: su función social.

\section{Resultados y discusión}

Para considerar el concepto de función social de la argumentación, se toma como referencia los aportes de Jauss (1987), fundador de la Teoría de la recepción, quien plantea una definición de la función social de la literatura, en donde manifiesta que la experiencia del lector, es decir, la práctica y los modos en que el lector se pone en contacto con el universo de la lectura y la escritura en un contexto de veloces transformaciones culturales y tecnológicas, entra en el horizonte de expectativas de su praxis de la vida, prefigurando o cambiando su concepto de mundo. Parece entonces, que esta función social de la literatura aplicaría también para comprender las dimensiones de las prácticas de argumentación. Desde el punto de vista de Jauss, se entiende que la literatura incide en las perspectivas de las personas en tanto los hace distintos con respecto a sus actuaciones y participaciones sociales. Eso mismo se puede plantear frente a la argumentación en cuanto es una práctica inserta en la vida de las personas que permite, entre otros aspectos, conocer el mundo, en tanto se informa y comunica, sacar provecho de las ideas de las otras personas, ejercer el espíritu crítico y democrático, o sea, permitir ser mejores individuos.

En este sentido, la enseñanza de la argumentación en la escuela debería admitirse en los programas académicos desde los primeros grados de la básica primaria, ya que esta permite a los estudiantes el acercamiento a un pensamiento crítico y divergente desde tempranas edades, el cual es necesario ir consolidando porque, más tarde, les servirá para efectuar una acertada participación ciudadana, capaz de transformar estructuras sociales para el bien común. Una argumentación bien concebida y realizada por los estudiantes es, quizás, un arma potente que permita rebatir o cambiar idearios arraigados en la sociedad por otros mejores.

\section{El carácter social de la escuela en la ruralidad}

La escuela en la cual tuvo lugar esta experiencia investigativa fue en el Centro Educativo Rural Montecristo en el municipio de San Juan de Urabá, departamento de Antioquia. Esta escuela debido al contexto en donde se encuentra localizada es el único centro de formación regular que existe en la comunidad, allí convergen niños de la misma vereda, de fincas equidistantes y de otras veredas. También es un lugar que sirve de asiento para llevar a cabo las reuniones comunitarias de carácter formal en donde se debaten ideas y se llega a acuerdos, lo que permite generar procesos reflexivos en torno al bienestar de la comunidad y de ella 
misma. Estos son discursos que admiten la llegada de ideas externas cargadas de subjetividades y de experiencias de la vida en la ruralidad, formas de interacción, discursos acerca de las riquezas y también las ausencias en la comunidad. Estas son razones para decir que en el entorno rural prima un estilo de vida concreto, con una identidad propia y un estilo de participación característico.

Por este carácter diverso en apreciaciones, participaciones y sentidos que se dan en la vereda, la enseñanza se debe gestar a partir de la identificación de raíces culturales y características propias del entorno, tales como los sentidos de las fiestas de la vereda, el porqué de las costumbres arraigadas, la valoración de fauna y flora del contexto, entre otros, que se convierten en potencialidades de la propia realidad y no de esquemas ajenos y proyectados desde fuera. Además, en este contexto el docente es visto como la persona que aporta al conocimiento. Su discurso es respetado y valorado como tal, aspecto que favorece al liderazgo y desarrollo de sus propuestas educativas, las cuales deberían ser viables y pertinentes para el mejoramiento de vida de las personas del contexto.

Según lo anterior, Gallardo (2011) expresa que «la escuela de contexto rural puede llegar a convertirse en un interesante «laboratorio de renovación escolar» [...] puede estar en condiciones de mostrarnos características particulares que hacen de ella un modelo de trabajo pedagógico y social [...] lo que podríamos denominar un modelo educativo de futuro» (p. 8); es decir, que la escuela rural representaría ese escenario sugestivo de experimentación y revisión de cualquier reforma educativa que desee partir, no de teorías psicopedagógicas o de motivaciones esencialmente económicas, sino de las propias necesidades y fortalezas reales de los estudiantes y de lo que circula en su entorno. Desde esta perspectiva, se entiende que la escuela rural podría llegar a desempeñar una función social, si tiene en cuenta el desarrollo de sus propias potencialidades y el óptimo aprovechamiento de sus recursos y posibilidades. Los cambios que demande serán posibles, además, solo con el aporte comprometido y responsable de los profesores, lo que permitiría, entre otras cosas, que los estudiantes puedan ejercer su participación en la cultura.

Sin embargo, sería necesario revisar hasta qué punto la escuela rural permite ejercer esta participación en la cultura. Considerando el planteamiento de Pérez (2004) en donde deja ver algunas reflexiones en torno a las funciones de la escuela que, en el campo del lenguaje, implican una orientación que va más allá de la pedagogía y la didáctica y se sitúan en el ámbito político, en el contenido de la participación amplia y efectiva de los ciudadanos, es decir, en el ejercicio de la consolidación de una democracia. La escuela rural, en este sentido, debería abrir el espacio para que dentro de ella se gesten prácticas socioculturales que orienten, de manera consciente y reflexiva a los estudiantes, teniendo en cuenta aspectos como su experiencia, creencias y concepciones para permitir una convivencia armónica, participativa y constructiva.

Se puede considerar entonces, desde esta parte, que el lenguaje (Pérez, (2004) se refiere a la lectura y escritura específicamente) es producto de la cultura en un término amplio y político, el cual brinda un posicionamiento de la identidad de la persona, una forma distinta de relacionarse con la cultura y con su acumulado de sentidos, es decir, formas distintas de construir mundos. Se podría decir, entonces, que el contacto con la argumentación posibilita esta 
intencionalidad del lenguaje porque facilita su uso de manera crítica y reflexiva, importante para ejercer ciudadanía, participar de manera consciente y propender por el bien común de las demás personas. En este aspecto, vale resaltar que

la escuela (rural) por su naturaleza social y como espacio de múltiples determinaciones, interacciones, intereses y expectativas, es un ámbito en el que el margen de autonomía efectiva para la acción con que contamos los docentes es muy amplio, debido, entre otras cosas, a que no existe una, llamémosla "ingeniera pedagógica y/o didáctica" que haya logrado, afortunadamente, estandarizar los procesos educativos (Pérez, 2004, p. 81).

De acuerdo con lo anterior, la escuela rural está llamada a promover, orientar y desarrollar planes académicos basados en nuevas estrategias que fomenten el pensamiento crítico de sus estudiantes. Debe prepararlos para que enfrenten, entiendan y resuelvan adecuadamente, los problemas concretos de su contexto o de otros a donde emigran, como las ciudades, que obstaculizan el mejoramiento de sus condiciones de vida. En otras palabras, su importancia radica en el aporte efectivo que debe hacer a la formación de sus estudiantes para que estos tomen parte activa y responsable en la vida social, económica y política de su comunidad, región o país. El desarrollo de las capacidades argumentativas en los estudiantes podría contribuirles en su orientación social y la escuela rural posee todas las condiciones para ello; es decir, los diversos contrastes que presenta, los diferentes intereses y formas de interacción que favorecen su puesta en escena.

\section{Acerca de la función social de la argumentación en la educación básica rural}

Tradicionalmente se ha considerado a la argumentación como la forma más destacada de organizar el discurso, tanto de manera oral como escrita, con el fin de conseguir la validación de las ideas $u$ opiniones expuestas, es decir, permite aportar razones para defender una tesis, además de ser el discurso de la controversia, por excelencia. Según estas circunstancias, las prácticas de enseñanza de la argumentación pueden llevarse a cabo siguiendo un manual de normas que son aprendidas casi de memoria para que el estudiante «pula» su enunciado, de tal forma que permita la aceptación de un destinario y logre persuadirlo, acciones que se distancian de un enfoque sociocultural.

De acuerdo con ese enfoque normativo, sería recomendable direccionar las prácticas de enseñanza de la argumentación en la escuela hacia hábitos prescriptivos y tradicionales, tales como el planteamiento de una tesis u opinión, la justificación mediante argumentos, razones o contraargumentos y una conclusión o punto de vista en sus producciones argumentativas, tanto orales como escritas. A su vez, hacer énfasis en los tipos de argumentos, recursos y estrategias de argumentación, los cuales pueden ser de autoridad, de causa-consecuencia, de ejemplificación o mediante ejemplos, de generalización, de analogía o semejanza, de comparación, de refutación, de ironía, pregunta retórica y concesión. Este proceso, que ha caracterizado las prácticas tradicionales de enseñanza de la argumentación, se ilustra mejor en las figuras $\mathbf{1}$ y 2. 
Figura 1. Estructura de un texto argumentativo.

\section{Estructura de un texto argumentativo}

\section{Introducción}

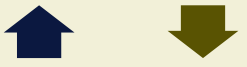

Planteamiento de tesis o hipótesis

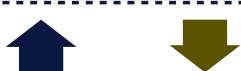

\section{Cuerpo argumentativo}

Exposición de las ideas y formulaciones de hipótesis Argumentos demostrativos (válidos)

Refutaciones de objeciones

Confirmación de la tesis

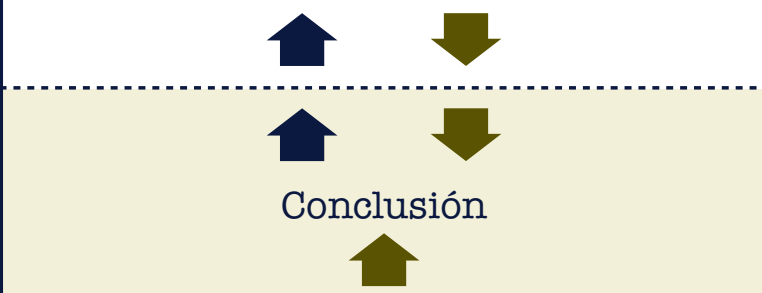

Síntesis de las ideas expuestas y demostrativas Aportaciones y sugerencias Nuevas propuestas deducidas
Como se puede observar en la Figura 1, la argumentación tiene una estructura lógica, basada en lo preceptivo del discurso en donde se evidencian tres partes: la introducción, el cuerpo argumentativo y la conclusión. Básicamente en la parte introductoria se hace un planteamiento de la tesis que se quiere demostrar, el cuerpo del trabajo hace referencia a la exposición de los argumentos para comprobar la tesis planteada y la conclusión se refiere a la síntesis de la argumentación y el aporte y sugerencia a nuevas propuestas deducidas.

Desde el modelo argumentativo de Toulmin, presentado por Rodríguez (2004), se muestra también una estructura básica de la argumentación, desde un punto de vista lógico. Según este modelo, toda argumentación, sin importar el tipo, contiene las siguientes partes (Figura 2):

Fuente: Sánchez, 2006, p. 382.

Figura 2. Modelo argumentativo planteado por Toulmin.

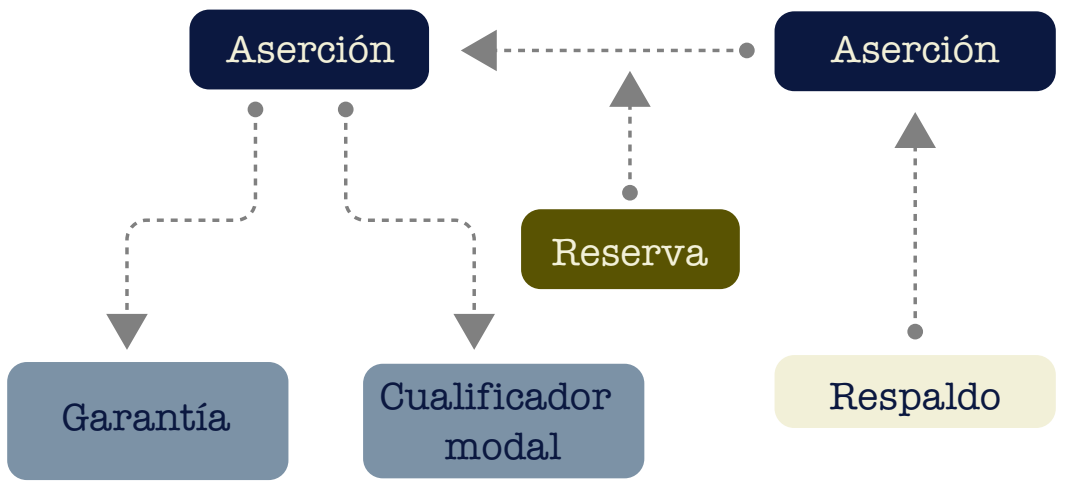

Fuente: Rodríguez, 2004. 
De acuerdo con este esquema, la aserción corresponde a la tesis que se va defender, a demostrar y sostener ya sea de forma oral o escrita, lo cual viene a ser el propósito de toda argumentación; la evidencia aporta informaciones o datos a la tesis para mantenerla y demostrarla; la garantía evalúa si tanto la inserción como la evidencia son eficaces y suficientes para los propósitos de la argumentación; el respaldo puede estar constituido de datos de autoridad o basados en creencias consolidadas socialmente, en estudios científicos, publicaciones o textos que apoyan la garantía, es decir, los fines argumentativos; el cualificador modal por su parte, especifica el grado de certeza, los términos, la fuerza de la aserción y las condiciones que la limitan; y, finalmente, la reserva se constituye en un espacio de la argumentación para mencionar las debilidades que se tienen acerca de la temática y la transformación que hace la persona que argumenta a su conveniencia. Se podrán decir que son conclusiones alternas.

Sin embargo, Bermejo (2003) considera que más que un conjunto de prescripciones y estructuras lógicas, «la argumentación resulta ser un instrumento fundamental, no solo de la reflexión teórica, sino incluso de nuestra forma de vida» (p. 106); es decir, toma además otra perspectiva más dialógica y menos acartonada que hace posible la convivencia y el encuentro con otras personas, desmontándola un poco de su carácter mecánico de conocimiento como se percibe en las figuras anteriores.

Esto da fuerza para considerar que, desde la educación básica rural, la enseñanza de la argumentación se debería orientar desde una concepción que permita insertarla en la mayoría de las situaciones cotidianas del estudiante, considerarla también como un diálogo con el pensamiento del otro, partir de la relación, interacción o de la propia confrontación de ideas con el otro por medio del lenguaje en donde hay un compartir de experiencias, que puede ser desde la experiencia de los demás o de la experiencia de la alteridad en la conversación. Esto se evidencia, por ejemplo, en las interacciones que tienen los estudiantes en los grupos de trabajo en el salón de clases, cuando van a comprar a la tienda escolar y establecen diálogo con personas en ese sitio, en las cartas que redactan, en los permisos que piden para ausentarse de las clases o de la escuela, cuando solicitan al rector espacios para alguna celebración, en fin, la argumentación atraviesa todos los discursos que promueven los estudiantes en sus eventos comunicativos.

De acuerdo con lo expresado, algunos docentes del Centro Educativo Rural Montecristo compartieron sus experiencias en las participaciones que hicieron en los grupos de discusión realizados en esta investigación, las cuales tratan de conversaciones e interacciones que sostienen con sus estudiantes. En ellas se puede notar que estos no llegan a la escuela como un «disco duro» nuevo, sin información; por el contrario, llegan a la escuela a compartir sus experiencias, «cargados» de discursos que se forman en su cotidianidad. El docente como tal, debe saber dirigir estos discursos hacia prácticas formales en donde se utilice el discurso argumentativo en este caso. Unos docentes decían:

El lunes yo les pregunté si por qué no habían hecho la tarea y me dijeron que se habían ido para un baile porque los papás habían ido al baile y ellos no se iban a quedar solos [...]; ellos vienen contando cositas de la casa, siempre me vienen con una cosa que: ¡Ay, seño! mi mamá se tuvo que ir para Apartadó por mi hermanito porque mi papá le pegó [...]; Seño, no 
me compraron nada con mi platica de Familias en Acción porque mi mamá pagó la luz con eso (Docente, grado tercero).

La mayoría de mis alumnos comentan lo que hacen por las tardes, sus paseos, sus atardeceres, cuando van al río. Yo los escucho, yo no les digo que no. Que si se roban unas guamitas, unos mangos, las patillas [...] Casi no hablan de que si hicieron la tarea sino lo que hacen: que fulano me empujó [...] Poco, poco cuentan lo que pasa en la casa (Docente, grado quinto).

Este tipo de discursos muchas veces pasa desapercibido por los docentes. Para la práctica de enseñanza de la argumentación, este compartir de ideas permitiría brindarle herramientas al docente para formar situaciones reales de argumentación dentro del salón de clases, es decir, prácticas socioculturales pertinentes. Se podría armar una situación comunicativa de este carácter enfocada en las posiciones de los estudiantes en lo correspondiente al maltrato de la mujer o del porqué no sería apropiado gastarse los recursos destinados para la educación de los niños. Este tipo de situaciones fomentaría los propósitos argumentativos.

Insertar estos tipos de discursos reales de los niños en las aulas de clases resultaría una labor loable en el campo de la enseñanza, porque esto permitirá al docente, en definitiva, crear situaciones comunicativas auténticas cargadas de subjetividades y experiencias de sus propios autores. Situaciones que serían insumos imprescindibles para comenzar a trabajar las prácticas de la argumentación desde una perspectiva sociocultural. Sin embargo, estos discursos no hacen escala en la esfera de la enseñanza, en este caso, en las prácticas de enseñanza de la argumentación, y pasan inadvertidos y son tomados como pequeños relatos, de forma fraccionada, que no producen efecto en la configuración didáctica, ejercicio íntimo del docente. En pocas palabras, el contexto rural aporta pocos recursos físicos al terreno de la enseñanza, pero los recursos discursivos reales son abundantes y diversos. Es tarea del docente saber aprovecharlos y ampliar la delimitación del lenguaje en las prácticas de enseñanza en estos entornos.

Sin embargo, el abordaje de la argumentación en la básica primaria rural es una temática que requiere más atención. Por un lado, está el desconocimiento, en gran parte, de las implicaciones sociales que las prácticas de enseñanza de la argumentación estriban; y por otro, está la concepción que se tiene de ella como una práctica basada en la prescripción que se limita a la comprensión y producción de textos, los cuales presentan una estructura rígida y convenida tal como se mencionó anteriormente. Asumirla de este modo sería ocultarle su carácter dialógico que permite una acción comunicativa recíproca. En relación, Perelman (1999) señala que «se dejan de lado las posibilidades que los niños manifiestan cuando se comunican y donde ponen en funcionamiento diferentes modalidades en la organización de su discurso. Ellos narran, describen y argumentan» (p. 1). En ese sentido, la argumentación es un discurso que tiene presencia en la cotidianidad del niño, lo cual permite su interacción; es la escuela la encargada de formalizar esos discursos. Al respecto Hurtado y Chaverra (2013) plantea que:

Todo lo que sucede en la escuela tiene que formar; pero, para que esto ocurra, los directivos y el equipo docente deben pensar en las diferentes experiencias formativas y promoverlas para que los estudiantes, toda vez que 
la lectura, la escritura y la argumentación se desarrollan con prácticas mediatizadas por los profesores y con intenciones pedagógico-didácticas claras (p. 11).

La escuela, entonces, debería comenzar a estructurar sus programas curriculares hacia propuestas de trabajo pedagógico que privilegien el proceso formativo de la argumentación en sus estudiantes. Que desde los primeros grados de la básica primaria se potencie este tipo de discursos y no dejarlo a la deriva o esperar que en los grados superiores de la secundaria se les comience a hablar de argumentación a los estudiantes o se aborde su enseñanza de manera consciente y sistemática. La escuela debe posesionarse en su rol formador basado en el conocimiento, la construcción y ejecución de estrategias de forma diferente para fortalecer la creatividad y la crítica.

Desde el concepto de participación en Kalman (2003) se podría considerar esta propuesta de trabajo pedagógico. La escuela rural por ser uno de los principales espacios en donde se gestan prácticas socioculturales debería brindar a los estudiantes la posibilidad de participar de ellas en su uso con personas que las conocen y utilizan. Además, representa, ese «laboratorio» de renovación escolar. La misma autora afirma que «la participación se refiere al proceso de intervenir en actividades sociales, así como las relaciones que se establecen entre los diferentes actores» (p. 43). En el caso particular de la enseñanza de la argumentación, el docente en la ruralidad debería insertar en ella el uso consciente y adecuado del lenguaje argumentativo a través de la participación de los estudiantes en prácticas socioculturales como por ejemplo, elaborar una carta al alcalde solicitándole una obra en la comunidad o crear la situación de diálogo con personas de la comunidad con fines argumentativos, en donde utilicen este tipo de discursos para acceder a diversos propósitos comunicativos. En otras palabras, tratar de manera formal los usos no escolares de la argumentación de los niños para que poco a poco tomen conciencia de su proceso comunicativo y puedan llegar a la consolidación de discursos más pertinentes y eficaces.

Este concepto de participación es importante a la hora de promover el discurso argumentativo dentro del aula de clases; en ese sentido, la práctica de enseñanza debe provocarla. Esta se facilita por el conocimiento que posean los niños acerca de las diferentes temáticas y problemáticas de la realidad, y por la familiaridad que tienen con los debates. Estos espacios de interacción lingüística deber ser promovidos por el docente a partir de un enfoque pedagógico- didáctico que los motive a la participación; por el contrario, un enfoque centrado en la exposición permanente del profesor que coarte las posibilidades de expresión de los estudiantes, impide que emerja una argumentación acertada.

En sintonía con lo anterior, a diario se observa a los niños participando en su contexto real. Ello ocurre cuando se es testigo de variados eventos comunicativos en los que utilizan, sin que se logren evidenciar de manera consciente, variadas formas argumentativas para adquirir sus propósitos. Entonces ¿por qué no vincular este concepto de participación en nuestras prácticas de enseñanza de la argumentación?; si, como se dijo, la argumentación permite que los estudiantes entren en contacto con sus compañeros a través de sus discursos para defender sus ideas, para ponerse de acuerdo en una acción común o para confrontar opiniones diferentes, lo cual es socialmente importante para los chicos porque los convierte en seres activos de la sociedad. El hecho de 
confrontar diálogos, participar con sus ideas, comunicarse, entablar acuerdos los inscribe como participantes de la dinámica social y en constructores de textos argumentativos; entonces ¿por qué no hablar de la función social de la argumentación en la escuela primaria, específicamente en el contexto rural?

A propósito de lo anterior, la argumentación tendría una importancia enorme en la vida social. Se utiliza entonces para justificar nuestros pensamientos o nuestros comportamientos, para comunicarnos, ejercer ciudadanía, persuadir a los demás de nuestros puntos de vista, influir en el comportamiento de los otros, como base para la toma de decisiones. Al respecto, dos docentes participantes de uno de los grupos de discusión en esta experiencia investigativa refieren que, para ellos, la argumentación cumple una función social en el contexto de la escuela rural. Esto ocurre cuando por medio de ella, los niños son capaces de

[...] respetar el punto de vista de los demás, saber hablar en público, saber expresarse. Que el niño tenga autonomía, que cuando pase al bachillerato sepan defender sus criterios, [...] ellos deben explorar la conversación. Argumentar de cualquier cosa, sin temor a equivocarse. Que puedan conversar con otras personas, dialogar en grupo (Docente, grado quinto).

En el campo, para nosotros los docentes es muy importante de que ellos interactúen, de que manejen y desarrollen oratoria, de que aprendan a ser argumentativos [sic] (Docente, grado primero).

Estos discursos dejan ver que, en el contexto rural, la enseñanza de la argumentación se vislumbra desde una función social que impacta fuera de las aulas de clases. Conversar con otras personas, defender sus posturas sin temor a la equivocación o desarrollar efectivamente la oratoria son condiciones de una función social. En este contexto, lograr que los niños de los primeros grados de básica primaria se integren o interactúen con los demás miembros de la comunidad por medio del discurso es una acción eficaz y pertinente del docente si tenemos en cuenta que son pocas las ayudas que reciben de los padres de familia para el fortalecimiento de las capacidades argumentativas. No obstante, una concepción de función social para la argumentación de este talente es muy reducida e instrumentalista para estos docentes. Más bien, hablar de tal aspecto de una forma más deliberada sería afirmar que las prácticas argumentativas de los niños también permiten perpetuar su identidad, fortalecer su carácter crítico y la creatividad que les permita promover y preparar el terreno para un cambio social real, adaptarse a la vida social, dentro y fuera de la escuela, a ejercer control social sobre las normas establecidas, saber seleccionar los miembros de una sociedad o disminuir las diferencias sociales.

Todos los discursos externos de los estudiantes llegan al aula de clases cargados de sentidos, subjetividades, vivencias y sueños que se van construyendo en el más vasto ideario infantil, pero que se entorpece con la encrucijada del lenguaje que circula en la escuela. Es el docente el encargado de apropiar tales discursos y recrear situaciones comunicativas auténticas que permitan su participación y que se logre vislumbrar así, la función social de la argumentación. De esta forma tendrá sentido la enseñanza en el área del lenguaje.

Tomando como referencia el planteamiento de Fernández (2006), es por medio del diálogo que la argumentación alcanzaría, de forma 
adecuada, un nivel comprensivo y el acercamiento a una confrontación de ideas frente al discurso del otro, con el propósito de llegar a establecer acuerdos y formas de convivencia, imprescindibles en la vida social. La argumentación permite que una comunidad que se relaciona a través del diálogo, conozca de los idearios de los demás personas, de sus formas de vivir y sentir, de sus necesidades, de sus ritualidades. La argumentación, de este modo, sería una estrategia trascendente para la construcción de una sociedad más culta y democrática que privilegie el tratamiento de la problemática social por medio del discurso, en donde la fuerza de la argumentación es la mediadora en la interacción entre las personas.

Por otro lado, desde el enfoque sociocultural planteado por Martínez (1999) se hace énfasis en la noción de apropiación y el carácter social del lenguaje como característica que le aporta en la consideración de una función social de la argumentación. La «comprensión del pensamiento y de su relación con el habla, así como otros fenómenos implicados en la vida social del lenguaje tales como «voces», modos de discurso, lenguaje social y dialogicidad» (p. 18), podrían entrar en relación con la argumentación desde el plano de la praxis del discurso. En el campo de la educación básica primaria rural, especialmente, esta función social muchas veces pasa desapercibida por el docente. Es más, resulta hasta agraviante utilizar la palabra «argumentar» en los primeros grados de básica primaria dado que los docentes no la consideran apropiado desarrollarla.

En los Estándares Básicos de Competencia del lenguaje (2006), que es el documento público que regula la enseñanza del área de lenguaje en nuestro país, se establece en varios de sus subprocesos que se deben enfatizar, desde los primeros grados de la básica primaria, en las posibilidades que brinda lenguaje argumentativo en los estudiantes. En el contenido de este documento se menciona que el niño, en estos grados, estará en capacidad de exponer y defender sus ideas en función de la situación comunicativa, elaborar y socializar hipótesis predictivas acerca del contenido de los textos, comentar programas de televisión, interactuar con medios de comunicación masiva, participar en la elaboración de guiones para teatro de títeres, leer diferentes clases de textos.

Todos estos subprocesos mencionados en el párrafo anterior corresponden a las primeras aproximaciones a un lenguaje argumentativo. Entonces no sería apropiado decir que en la básica primaria al niño no se le debe poner en contacto con la argumentación, que en sus situaciones comunicativas no tenga presencia el lenguaje argumentativo. Los estudiantes podrían interactuar con los demás compañeros, participar, ser críticos ante la información que circula a su alrededor usando este discurso de manera consciente. No obstante, Cotteron (1995) señala que «numerosos profesores consideran aún que la enseñanza de la argumentación debe reservarse a los alumnos de secundaria obligatoria y no hay por qué dirigirse a los alumnos de primaria» (p. 79). Sin el ánimo de dejar de lado estas reflexiones que se tejen sobre la argumentación en el campo educativo y su lugar fundamental dentro del conocimiento teórico, también hay que considerar que ésta cumple una función social, como ya se dijo, y que implica que se aborde, no solo como instrumento garante del conocimiento sino también como discurso práctico, vivencial y activo basado en la experiencia de los estudiantes. 
En consecuencia, estimular las posibilidades de un lenguaje argumentativo en la básica primaria requiere de la elaboración de propuestas curriculares y/o configuraciones didácticas que desarrollen prácticas de enseñanza enfocadas a promover la participación en prácticas socioculturales auténticas en donde los estudiantes utilicen la argumentación para fines comunicativos, para dialogar con el pensamiento del otro. Al respecto, Hurtado y Chaverra (2013) plantean que

estimular la capacidad argumentativa en los niños requiere de la construcción y el desarrollo de situaciones comunicativas que convoquen su participación, uso de la palabra, expresión de sus puntos de vista, capacidad para tomar decisiones; en suma, los niños deben interactuar en prácticas y situaciones discursivas en las que sea necesaria la argumentación (p. 19).

Para finalizar esta experiencia investigativa, a medida que la sociedad se construye e irrumpe en nuevas tendencias comunicativas, la función social de la argumentación adquiere mayor relevancia en tanto al desarrollo del pensamiento, la autonomía, la participación y la toma de decisiones. Se trata entonces de ponerla «al alcance de los niños» y encontrarla en los diálogos que construyen, en su interactuar con el contexto escolar, en los textos que realizan, en las lecturas de los cuentos, es verla como «algo» próximo a su cotidianidad. En realidad, se trata de verla más allá de un conjunto de prescripciones, porque no podemos negar que la argumentación obedece también a unas reglas. Vista de esta forma, estaremos más cercanos y conscientes de su función social.

\section{Reflexiones finales}

\section{de la investigación}

Una experiencia investigativa en educación enfocada en el contexto rural como esta permite orientar procesos de reflexión permanente hacia los modos de llevar a cabo la enseñanza. Las consideraciones expuestas en este apartado abren caminos para continuar pensando sobre el estado de la enseñanza, especialmente en lo relacionado con la argumentación en la ruralidad. Además, permite el acercamiento a nuevos horizontes conceptuales y líneas de sentido para construir propuestas de renovación de la propia práctica en estos contextos. A continuación, se presentan algunas consideraciones finales:

1. La comprensión de las prácticas de enseñanza de la argumentación, desde un enfoque sociocultural, tiene unas implicaciones de orden didáctico en la escuela rural, cuyas configuraciones parten de la experiencia y la cotidianidad de los estudiantes con el contexto. En esta dirección, vale la pena empezar a explorar y a diseñar estas prácticas de enseñanza desde una perspectiva que tome en cuenta los discursos que los estudiantes comparten con las otras personas en su diario vivir, más que como herramienta de conocimiento centrada en la producción y comprensión de textos escritos con una estructura rígida y convenida. No quiero decir que esto no sea importante dentro de su enseñanza, sino que un enfoque de este estilo disminuye las posibilidades de producir un contexto de comunicación espontáneo y natural donde haga presencia el discurso argumentativo. 
2. El diseño de configuraciones didácticas, en el contexto rural, en el nivel de básica primaria, enfocadas a la enseñanza de la argumentación, tomando como guía una perspectiva dialógica, permite a los estudiantes una mejor apropiación del lenguaje y las posibilidades de exploración de las capacidades de un pensamiento crítico; es decir, propicia una mayor participación y compromiso entre los estudiantes en el proceso de apropiación del conocimiento y búsqueda de coherencia desde el desarrollo consciente de sus propias capacidades discursivas y la aceptación de puntos de vista divergentes que incidan en una formación autorreguladora y emancipadora que permite fortalecer sus capacidades de tomar posición frente a los fenómenos sociales que circulan a su alrededor.

3. Al desarrollar prácticas de enseñanza de la argumentación, se debe tener en cuenta la adecuada selección de los recursos. No solamente desde el texto escrito y su análisis se exploran las posibilidades de un lenguaje argumentativo, también existen otras formas que permiten un acercamiento claro y pertinente a este discurso, por ejemplo, desde el análisis de una noticia observada en la televisión o insertar a los estudiantes a verdaderas prácticas socioculturales de la argumentación, como una conversación con algún miembro de la vereda o del municipio o la redacción de una carta. Las prácticas de enseñanza de la argumentación, en El Centro Educativo Rural Montecristo, se han desarrollado dentro del marco de la enseñanza tradicional, desde un enfoque que no contempló mucho el diálogo respetuoso y civilizado de las ideas. Más que todo se hace intentos por desarrollarla desde un currículo técnico centrado en el carácter instrumental de la lectura y la escritura y el seguimiento de la política de Estado sin que se logren vincular los discursos reales de los estudiantes a la práctica de su enseñanza.

4. Las dificultades específicas que presentan los estudiantes de la básica primaria en este contexto rural para llevar a cabo un leguaje argumentativo obedecen, quizás, a la falta de claridad en cuanto a su enseñanza por parte de los docentes, es decir, no hay claridad en el enfoque de enseñanza. Algunos piensan que argumentar es solo exponer frente a los compañeros o poner a los niños a narrar historias. Se debe partir entonces desde un enfoque que permita la enseñanza de la argumentación a partir de acciones reales, de la valoración de los discursos de los estudiantes y sus modos de pensar. Integrar cada vez más el contexto con las actividades que se desarrollan en la escuela para fomentar acciones discursivas que acerquen a los niños a verdaderas prácticas argumentativas.

Para concluir, en estas consideraciones, se desea hacer una invitación extendida a profesores, investigadores y maestros en formación a continuar con indagaciones relacionadas con la enseñanza de la argumentación en contextos rurales, por ejemplo: cómo argumentan los niños en su contexto real y cómo lo hacen en la escuela, qué tanto incide la poca argumentación de los niños en la ruralidad en su rendimiento académico, qué formas de intervención en las configuraciones didácticas serían más pertinentes para explorar las posibilidades argumentativas de los estudiantes, en fin; son muchos los caminos que faltan por explorar y recorrer. Lo que sí es claro es que investigaciones en este orden son pertinentes y necesarias porque le dan carácter epistemológico a las prácticas de 
enseñanza, al hacer de la acción, de la experiencia, una fuente de teorización; investigaciones que posibilitan avanzar en la construcción de configuraciones didácticas desde la valoración de lo que ocurre en el contexto.

\section{Referencias}

- Bermejo L. (2003). Filosofía y retórica: el lugar de la teoría de la argumentación. Revista de Filosofía, 30, 105-115. Recuperado de: https://bit.ly/2K5rfc2

- Cotteron, J. (1995). ¿Secuencias didácticas para enseñar a argumentar en la escuela primaria? Comunicación, Lenguaje y Educación, 25, 79-94.

- Fernández, F. (2006). Conversación, diálogo y lenguaje en el pensamiento de Hans-Georg Gadamer. Anuario filosófico, 39, 55-76. Recuperado de: https://bit.ly/31g3DGN

- Gallardo G. (2011). La escuela de contexto rural: ¿de la diferencia a la desigualdad? Revista Iberoamericana de Educación, 55, 1-10. Recuperado de: https://bit.ly/2F9amJx

- Gascón, M. \& García, A. La argumentación en el Derecho, Algunas cuestiones fundamentales. Madrid: Editorial Palestra.

- Hurtado, R. \& Chaverra, D. (2013). Enseñanza de la argumentación en la infancia. Medellín: Universidad de Antioquia.

- Jauss, H. (1987). Historia de la literatura como una provocación a la ciencia literaria. México: Universidad Nacional Autónoma de México.
- Kalman, J. (2003). El acceso a la cultura escrita: la participación social y la apropiación de conocimientos en eventos cotidianos de lectura y escritura. Revista Mexicana de Investigación Educativa, 17, 37-66.

- Martínez, M. (1999). El enfoque sociocultural en el estudio del desarrollo y la educación. Revista Electrónica de Investigación Educativa, 1, 16-37.

- MEN. (2006) Estándares Básicos de Competencias en Lenguaje, Matemáticas, Ciencias y Ciudadana, Bogotá: Revolución educativa.

- Mendoza, A. (2015). La validez en los exámenes de alto impacto. Un enfoque desde la lógica argumentativa, Perfiles educativos, 37, Recuperado de https://bit.ly/31g6xLU

- Ochoa, L. (2005), Estrategia didáctica para el desarrollo de la argumentación oral en quinto grado de educación básica colombiana, Tesis de maestría no publicada, Universidad Nacional de Educación a Distancia, España.

- Perelman, F. (1999). La producción de textos argumentativos en el aula. Revista en el aula, 13, 2-11, Ministerio de Educación de la Nación, Argentina. Recuperado de: https://bit.ly/2IpPGOC

- Pérez, M. (2004). Leer, escribir, participar: un reto para la escuela, una condición de la política. Lenguaje, 32, 71- 88.

- Rodríguez, L. (2004). El modelo argumentativo de Toulmin en la escritura de artículos de investigación educativa, Revista Digital Universitaria, 5, 118. Recuperado de: https://bit.ly/2Xw349R

- Sánchez, J. (2006). Saber escribir. Madrid: Editorial Aguilar. 